\title{
Reducing uncertainty - responses for electricity utilities to severe solar storms
}

\author{
Charles Trevor Gaunt* \\ University of Cape Town, Rondebosch, Cape Town, South Africa \\ *Corresponding author: ct.gaunt@uct.ac.za
}

Received 3 May 2013 / Accepted 23 December 2013

\begin{abstract}
Until recently, electricity utilities in mid- and low-latitude regions believed that solar storms had no (or only insignificant) effect on their power systems. Then it was noticed that the onset of damage in several large transformers, leading to their failure, correlated very closely with the Halloween storm of 2003. Since then engineers have started to appreciate that a very severe storm could have serious consequences outside the high-latitude regions. There are many uncertainties in predicting the effects of solar storms on electrical systems. The severity and time of arrival of a storm are difficult to model; so are the geomagnetically induced currents (GICs) expected to flow in the power networks. Published information about the responses of different types of transformers to GICs is contradictory. Measurements of the abnormal power flows in networks during solar storms generally do not take into account the effects of the current distortion and unbalance, potentially giving misleading signals to the operators. The normal requirement for optimum system management, while allowing for the possibility of faults caused by lightning, birds and other causes, limits the capacity of system operators to respond to the threats of GICs, which are not assessed easily by the $N-1$ reliability criterion. A utility's response to the threat of damage by GICs depends on the expected frequency and magnitude of solar storms. Approaches to formulating a response are located in a system model incorporating space physics, network analysis, transformer engineering, network reliability and decision support and the benefits are identified. Approaches adopted in high-latitude regions might not be appropriate where fewer storms are expected to reach damaging levels. The risks of an extreme storm cannot be ignored, and understanding the response mechanisms suitable for low-latitude regions has the capacity to inform and reduce the uncertainty for power systems planners and operators worldwide.
\end{abstract}

Key words. geomagnetically induced currents - power systems - reliability - transformers - condition monitoring

\section{Awareness of a problem}

Complexity contributes to uncertainty. Large power systems are complex, comprising many components and operating under variable conditions to meet the constantly changing requirements of the customers for power.

Components of the power system fail for diverse reasons, including ageing, corrosion, lightning and human interference. Redundancy is provided to reduce the effects of component failure, but still some customers' supplies are interrupted when too many components fail and, in extreme situations, the collapse of a whole system causes widespread blackouts such as in North America and Italy in 2003 (Andersson et al. 2005) and India during 2012 (Ministry of Power 2012).

A system with sufficient redundancy is able to continue supplying the customers in spite of an unexpected component failure, and is said to be operating in a normal state. The reserve margin, a term mostly used to describe generation capacity, represents the redundancy available to make up for the loss of any component, termed a contingency event. When there is insufficient redundancy, the loss of one or more key components (generators, transformers and/or feeders) initiates interruptions. However, the real problem starts before the component or a combination of components is lost. External events, internal degradation and loading all contribute together to the likelihood that contingencies will exceed the compensation capacity of the system. Providing enormous redundancy is feasible but too costly. Therefore, system planners and operators need to find optimum conditions, continuously.

Planners and operators develop a good understanding of the most common problems and operating conditions. Less frequent phenomena, when external threats affect many network components at the same time, are less well understood, especially when the threat itself is virtually invisible and its region of influence poorly defined, as with geomagnetic disturbances.

Geomagnetic storms severe enough to disrupt power systems are uncommon and, until recently, were thought to have no effect on power systems in low-latitude regions. This is not really surprising, since the mechanisms leading to failure were relatively poorly understood. Investigations in South Africa during the 1990s, after the Quebec storm of 1989, found no evidence that geomagnetic storms were a problem. Both the power system and the effects of geomagnetic storms are complex and, with the hindsight of our present understanding, the conclusions of those investigations are not surprising.

The Halloween storm of October 2003 led to a different conclusion - that solar events could affect power systems in low-latitude regions. The conclusion that geomagnetically induced currents (GICs) could be a problem (Gaunt \& Coetzee 2007) was disputed. Subsequently research in other countries has shown that significant GICs are not limited to high-latitude regions (Liu et al. 2009; Torta et al. 2012). Initially the mechanisms of failures apparently initiated by GICs were not well understood and the failures that had occurred were attributed 


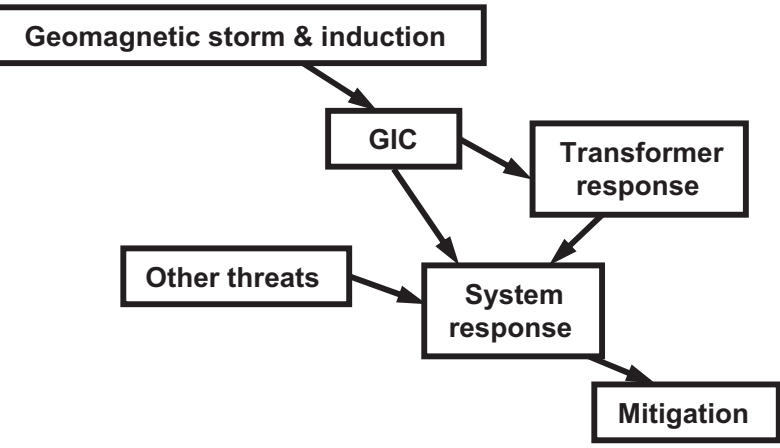

Fig. 1. Systems approach to GICs.

to various other causes. The situation is outside the normal experience of system planners and operators and cannot be represented by average conditions. The complexity of predicting geomagnetic disturbances (GMDs) and their effects on power systems create substantial uncertainty for electricity utilities (Thomson et al. 2010)

This paper presents the findings of some research undertaken since 2007 to reduce that uncertainty.

\section{Model of the system}

The system needing to be considered is greater than a power system. It includes those events on the sun that initiate GICs and extends to the possible responses of the utility. Figure 1 shows a model of the system used in our research.

The system has five sub-systems incorporating these elements:

Space physics includes the processes from disturbances on the Sun to the induction at Earth of the voltages that drive GICs in the power system.

Network analysis calculates the actual currents in the network's lines and transformers, including the GICs and the effects on the network of the transformer response.

Transformer engineering investigates the responses of the transformers to the induced currents, including the transient response to quasi-dc, the generation of harmonics that distort the power frequency sinusoidal voltages and currents, the processes of overheating and the degradation and failure of the transformers.

Network reliability and cost assessment considers the performance of the network as a whole, affected by other threats as well as GICs, and the likely damage cost associated with any operating condition.

Decision support for network planners and system controllers models the implications of alternative approaches to preventing or mitigating the system responses of physical and financial damage that can be caused by GICs.

\subsection{Space physics}

Space physics models the propagation of solar activity events through the heliosphere, their interaction with the Earth's magnetosphere, the resulting disturbances of the Earth's magnetic field, and the induced voltages that drive the GICs in electrical conductors like power lines.

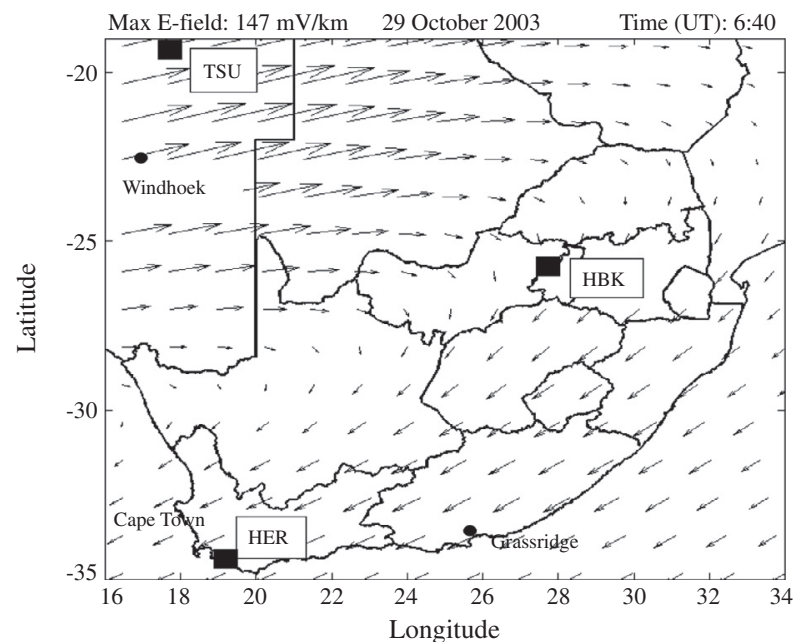

Fig. 2. Grid of interpolated horizontal electric field over Southern Africa on 29 October 2003 at $06: 40$, calculated using measured magnetic fields at three observatories.

The severity of geomagnetic disturbances is not represented by the size of the solar cycle peak. The magnitudes of the most severe disturbances in each solar cycle, measured (or for some earlier events estimated from other parameters) by the widely used 3-hourly index $a a$, do not vary much with the magnitudes of the solar cycles as measured by sunspot number. The peak sunspot number of the solar cycles when two of the most severe GMDs recorded (in 1859 and 1921) occurred were quite similar to that of the present solar cycle expected to peak during 2013. So, existing indices like the $a a$ index might not be suitable indicators of the severity of disturbances on power systems.

It is clear that several storms have been more severe, in terms of extended periods of large time derivatives of the geomagnetic field and the induced GICs, than the Halloween storm that is believed to have been the initiator of significant damage to transformers in the South African power system during 2003. It is now over 150 years since the extreme storm known as the Carrington event in 1859, and the likelihood of another similar or larger disturbance is of great interest to power systems engineers.

The frequency of extreme disturbances has been studied by various researchers (Thomson et al. 2011; Pulkkinen et al. 2012). Although probability distributions do not address the uncertainty of when severe and extreme events will occur, they do reduce the uncertainty in modelling the maximum likely stress on the power systems.

Building on work done in other countries, electric field interpolation techniques have been used to model the conditions in South Africa during GMDs. Figure 2 (Bernhardi et al. 2008) illustrates how non-uniform the field was at one stage of the Halloween storm and that it was closely aligned to the topology of the main transmission network. Similar maps at 2-minute intervals show the field was turbulent in respect of magnitude and direction for several hours. Clearly, early assumptions of a uniform plane wave model were quite inaccurate. Figure 2 shows three of the magnetic observatories in Southern Africa at Hermanus, Hartebeeshoek, Tsumeb and another is now operational at Keetmanshoop, South of Windhoek.

The severity and characteristics of disturbances in South Africa might reasonably be expected to be similar to disturbances at other locations of similar geomagnetic latitude, such 
C.T. Gaunt: Responses for electricity utilities to severe solar storms

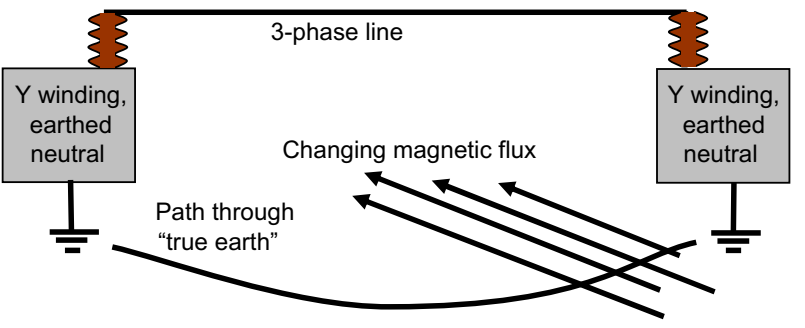

Fig. 3. Induction of GICs in the power transmission system.

as in North America, Europe, China and Australia. However, the expectation is coarse and does not take into account the time of day, the possible interaction between the equatorial ring current and polar electrojets during significant disturbances, and the different ground resistivity in different regions. Research on these aspects will be useful as a basis for comparing power system responses in the various regions, and further reducing uncertainty.

Space physics also contributes the models of induced current in the branches of the power system network connecting the nodes (substations) where transformers are installed with the neutral points earthed (grounded). GICs flow in loops illustrated in Figure 3 created by:

- the conductors of high voltage transmission lines, usually comprising two or more sub-conductors for each of three phases of the alternating current power system but providing effectively a single composite conductor for the quasi-dc GIC,

- the star- or $Y$-connected windings of the transformers at each end that have the neutrals earthed for the purposes of safety, operations control and reducing the cost of the transformers and

- the conductance of the Earth through which the current disperses widely. Although it is dispersed, the current is often represented as an equivalent current at a particular depth. The deep, dispersed path is often referred to as "true Earth" because its very low resistance is dominated by the localised electrical connections to it of the transformer neutrals in the substations.

The variation of the magnetic field, and thus the induced current, has a typical cycle time of minutes $(\sim 0.01 \mathrm{~Hz})$, which is very slow compared with the rate of change of voltage and current in the power system $(50$ or $60 \mathrm{~Hz})$. Therefore, power systems engineers often refer to GICs as being quasi-dc in their character, but it is important to realise that the currents do vary slowly and with different components of frequency. Since the effective depth (tens to hundreds of kilometres) at which the equivalent current flows in the "true Earth" changes with the effective frequency, the area of the current loop depicted in Figure 3 varies and the relationship between the magnetic flux change and the induced E-field is also not constant.

Of course, post-event calculations of field conditions and induced currents are useful for research, but the operations control staff of an electricity utility need real-time data and, preferably, forecasts of storm onset and severity about $6 \mathrm{~h}$ ahead. These models are not yet available. More accurate modelling based on measurements at the Lagrange point L1, giving about 20 min warning during extreme events, would be useful in reducing uncertainty.

\subsection{Network analysis}

Network analysis, based on models of GIC induced in the overhead lines of the transmission network, calculates the currents expected to flow in the substation transformers. It is well known that the magnitudes of the induced currents in the lines and transformers depend on the characteristics of the GMD, the resistivity of the ground and the network configuration (topology and conductor resistances). Until recently, network analysis has not taken into account the time delay that arises from the charging of a large transformer - an inductance - by the quasi-dc GIC, and the way the transformers' responses over several minutes to step changes of induced voltage effectively filter the prospective GIC (Oyedokun et al. 2013). As shown by the turbulent field modelling, the calculation of network and transformer currents is a dynamic process, and it is also influenced by the physical construction of the transformers. Three-phase 3- or 5-limb and single-phase transformers all respond differently to imposed GIC potentials, and the network analysis must take the differences into account.

New approaches to network calculations, from basic principles and based on mapping a disturbance to the response, are being attempted. Good agreement between calculated currents in transformer neutrals and measurements recorded during large geomagnetic disturbances in 2001, 2003 and 2004 has been achieved. Even small disturbances, such as have occurred several times already during the present solar cycle, produce measurable responses in the transformers. Reliable current measurements in the neutrals of many transformers in many parts of the network are needed to calibrate the calculations more accurately, and these are now being put in place. The new approaches, after testing with even small signals from minor GMDs, could successfully model more intense storms.

Network analysis also calculates the power flow in the system, essential for understanding the network's capacity to maintain acceptable voltage conditions. It is widely known that GICs flowing in transformers increase the non-active (reactive) power required from the system (Molinski 2002), explaining the unusual real and reactive power swings and voltage depressions observed during the Quebec storm (Kappenman \& Albertson 1990). More recent work has shown that a new definition and method of measuring apparent power and power factor is needed to allow potential voltage-instability conditions on a network to be interpreted correctly under conditions of distortion, unbalance and with dc components (Malengret \& Gaunt 2011, and companion papers). Laboratory-scale measurements and simulations have shown that the power factor, reflecting the effective capacity of a system to deliver power, is substantially lower than indicated by conventional measurements when dc components flow in transformers (Gaunt \& Malengret 2012). Since a recent NERC report on reliability concludes

The most likely worst case system impact from a severe GMD event and corresponding GIC flow is voltage instability caused by a significant loss of reactive power support simultaneous to a dramatic increase in reactive power demand (NERC 2012),

the new approach could help operators to identify the best system configurations.

\subsection{Transformer engineering}

The GIC component of the current flowing in a transformer disturbs the normal power-frequency operation of the magnetic 


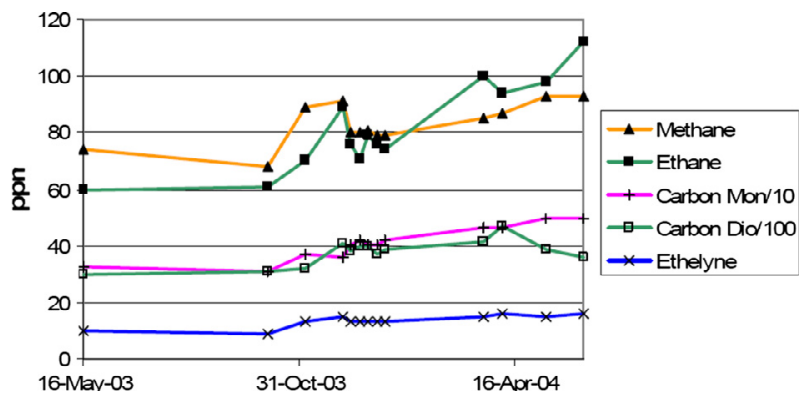

Fig. 4. DGA record of generator step-up transformer showing gassing starting end October.

circuit, causing core saturation, increasing the leakage flux and generating harmonic distortion and voltage unbalance. These effects in the transformers have the capacity to cause overheating and damage that depend on the design of the transformers and the load being carried, as well as on the magnitude, variation and duration of the induced currents.

The NERC report (2012) identifies the most likely impact of extreme GICs on power systems as voltage instability, but it also recognises the risk of damage to transformers and other equipment, and that restoration times for damaged equipment would be much longer than for a power system collapse. Power system collapse during extreme geomagnetic disturbances could protect equipment from damage but when disturbances are less severe, or in regions not so severely affected by an extreme event, the transformers and other equipment may be exposed for long periods to potentially damaging currents. It is difficult to predict completely the damage likely in a transformer because of the many variables, but continual progress is being made in the modelling of currents, the heating effects, transformer condition monitoring and fault investigation.

Although intense GMDs have been associated with physical melting of steel components of transformers (Kappenman \& Albertson 1990), the nature of damage in transformers at mid-latitudes is quite different. It is postulated there are many different routes to failure, according to the conditions in the transformer at GIC commencement. For example, in appropriate conditions, bubbles can form within a winding if leakage flux and eddy currents cause rapid heating and change the moisture equilibrium between the oil and paper insulation. Flux shunts on the core overheated by leakage flux can burn the oil, also generating bubbles that can be drawn into the winding by cooling oil flow. Bubbles are known to be sites for partial discharges that damage the paper insulation, and in a laboratory-scale experiment it was found that partial discharge could be turned on and off by very small dc currents injected through a transformer (Oyedokun et al. 2011). Alternatively, overheating is associated with sulphur deposition in some transformer oils, leading to a different mechanism of breakdown. Damage initiated by GICs might become self-sustaining, leading to progressive deterioration and eventually failure.

Several dissolved gas analysis (DGA) records from generator step-up transformers after the Halloween storm showed the sort of trends that would be expected from these kinds of failure mechanisms. Figure 4 illustrates an increase of gas content starting around the date of the storm. Despite unloading the transformer for a period and filtering the oil in other transformers, seven units with this characteristic increase in dissolved gases starting at the end of October continued to failure within one year. The failed transformers were not especially old, and most were considered to be around half life at their time of failure.

The interpretation of DGA records like the one shown in Figure 4 is time consuming and difficult, so the trajectories of some gases might even be omitted, especially when the damage caused by the GICs is not immediately evident. Therefore, the DGA measurements of the key gases (hydrogen, methane and carbon monoxide) associated with low-energy degradation of paper and oil have been combined in a triangular representation of the transformer health (Moodley \& Gaunt 2012). The lowenergy degradation triangle is similar in appearance to the Duval triangles already used in some utilities for transformer monitoring, but it focuses on insulation degradation at low temperatures. It is interpreted differently, since results in the left bottom vertex of the triangle represent a healthy transformer and movement away from this vertex indicates increasing degradation. The low-energy degradation triangle illustrated in Figure 5 clearly depicts the progress to failure of a 700 MVA GSU transformer similar to the one depicted in Figure 4. The analysis supports the conclusion that the Halloween storm contributed to this transformer's failure (Gaunt \& Coetzee 2007), but additionally a similar weaker response, not previously identified, appears to have occurred after the smaller storms of 2001. Although the unit was repaired and returned to service in May 2003, the GICs in October and November 2003 clearly accelerated a different failure after the Halloween storm.

The trend towards failure illustrated in this example represents only one of the failure mechanisms that occur in transformers. Other characteristic patterns have been identified, associated with different failure processes in other transformers.

This new tool for transformer condition monitoring is easily automated for on-line dissolved gas monitoring. In the past, transformer failure prediction has been very uncertain, both generally and when caused by GICs, but now can be substantially improved.

\subsection{Network reliability and cost assessment}

Transformers are not the only components in the network disrupted by the effects of GMDs. The distortion of the power frequency voltage and current by GICs in the transformers can affect capacitors, protection relays and the non-active (previously called reactive) power flow essential for network voltage stability. GPS-based communication and protection may maloperate or fail if the satellites lose lock as might occur during severe events. GIC warning systems dependent on Internetbased systems might similarly not perform reliably.

In addition, system operators need always to consider the effects of all other causes of coincident component and system failure. In South Africa the dominant external causes of faults on the main transmission system are birds, lightning, fires and pollution, together accounting for nearly $90 \%$ of all line faults (Minnaar et al. 2012). GICs are uncommon initiators of faults, although the effects may be widespread, making inappropriate the adoption of the usual reliability criterion of $N-1$ independent outage contingencies. Further, exposures of systems to GICs are of very limited duration relative to the life of the power system assets. Therefore, new techniques are needed to analyse the multiple contingency effects associated with both large power systems and the simultaneous exposure of many components to the threat of GICs.

Lightning, birds, fires and pollution all have distinct seasonal and time of day patterns. The demand on the system 


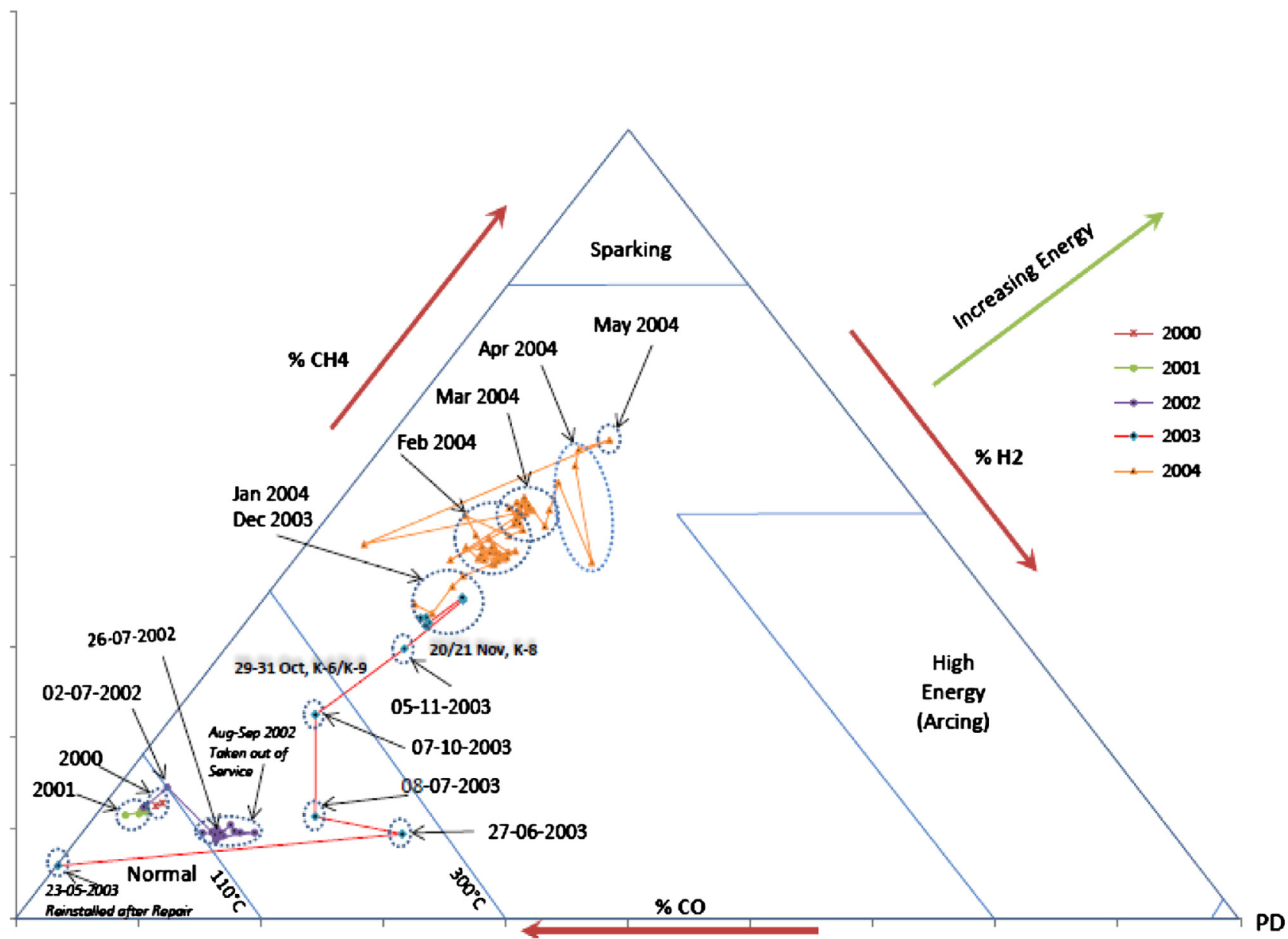

Fig. 5. Low-energy degradation triangle for transformer health assessment (Moodley \& Gaunt 2012).

exhibits similar patterns. Based on these properties, a timedependant probabilistic approach has been developed for reliability analysis. Defining four seasons and four times of day, the vulnerability of components to all adverse external threats is modelled by Beta probability density functions for each season- and time-period (Minnaar et al. 2012). A consistent approach can then be taken to Monte Carlo modelling of system failure for both planning and operations (Edimu et al. 2013). The approach copes effectively with the time-dependant correlation between threats and demand.

The costs of interruptions to customers can be applied to the output of the reliability (or failure) analysis. Surveys have identified that interruption costs also correlate with business activity levels according to the seasons and time of day and can also be represented by Beta probability density functions. The costs of interruptions at each busbar are aggregated for the customer mix. The aggregate cost is combined with the probability of failure at each busbar, allowing the total value at risk to be identified for the system configuration. The total cost is also a Beta probability distribution, from which singular values can be extracted for a given level of confidence or risk (Dzobo et al. 2012).

\subsection{Decision support}

The whole combination of the five sub-systems can be used for testing the cost-effectiveness of various approaches to preventing or mitigating the effects of GICs in power systems. The integrated approach is needed because the system is large and complex and characterised by uncertainty. Understanding the implications of any decision is beyond the scope of more simple, disconnected "solutions".

For example, the flow of GICs in a power system can be interrupted by installing series capacitors in the lines making up the network. Although this is costly, causes redistribution of GICs in circuits that are not blocked, and might introduce problems of sub-synchronous resonance, the expense is justified in regions where GICs are sufficiently common to justify the cost of protecting the assets. In low-latitude regions, such as South Africa, the solution could work, but cannot be justified financially, so alternatives must be considered. However, such alternatives have not been implemented widely, so there is little experience available locally or internationally to guide the decision-making. In the absence of that experience or other ways to find the best solutions to the problems of transformer failure and the threat of an extreme storm, a completely integrated approach to the whole system is needed.

An advantage of the modular design of the integrated system is that the modules can be developed in parallel. Further, as the ways of dealing with the complexity within each module are improved, the whole system becomes incrementally better at guiding the various decisions, including the final outputs for planning and operations.

\section{Benefits}

Another advantage of taking a systems approach to the threat of solar storms is that the whole is greater than the sum of the 
parts. The above descriptions of sub-systems show that the approaches have been conceived to feed into each other, so the whole system moves raw data from various sources towards decision support. At the same time, each process has outputs that feed into completely different systems. A few examples must suffice to illustrate the extra benefits derived from the research.

The work on the definition and measurement of power under non-ideal conditions was initiated by twin needs - to design power electronic controllers to correct power quality problems and connect renewable energy sources to the grid, and to understand better the distortion and performance of power systems in the presence of GICs.

The low-energy degradation triangle was developed to address specifically the uncertainty about the mechanisms of failure of transformers in our region. The need was evident because transformer failures were too numerous to be coincidental, yet could not be fully explained by what was known about GICs. The solution conceived to analyse what was happening turns out to be a tool suitable also for the early detection of low-energy degradation and warning of incipient failure. The tool can be incorporated, virtually unchanged, in standard programmes for transformer condition monitoring and asset management.

As knowledge of the transformer degradation processes improved, a better understanding was gained of the most relevant parameters of GMDs. Without interfering in the scientific research of space physicists, the definition of distinct applications has provoked new approaches for some of that research.

We determined that reliability analysis could be improved by adopting a 16-cell, time-based matrix of inputs to reduce the complexity of dealing with continuous functions in Monte Carlo simulations. At the same time, the output can be modelled as a probability to be interpreted with a level of confidence. The approach required classifying existing failure data in a new way, which has had spin-off already in identifying ways to improve transmission system reliability. The same time-dependant approach allows reliability analysis to be applied to long term planning and short term (6-h periods) operations control using closely related algorithms, common input data and consistent interpretation of the results of simulations.

Although developed to address specifically the uncertainties of high impact, low frequency GIC events, several of the techniques provide new tools suitable for extreme events other than those related to GICs, as well as for normal, everyday planning and control.

Finally, from the point of view of a university, the research has served as a vehicle for the intellectual training of a relatively large number of students, several of whom have already found responsible positions in industry.

\section{Implementation}

There is still a gap between having a plan or design and having a working project in place. The plan was conceived in 2004 and design has progressed according to the availability of resources - both funds and people. Many problems have been solved in the process. The work has also identified new possibilities for improvement in this project and applying the evolving techniques in other projects.

Completely new algorithms and tools have been developed and tested as far as is possible using limited data. Much of the work has already been published in various forms for critical review. However, the real benefits will only be realised when the design is put into use. We do not underestimate the effort required to ensure that every practical detail is working properly.

It is also recognised that implementation of a warning and mitigation system for responding to extreme geomagnetic events is not trivial. In addition to the algorithms, techniques and understanding already developed, there is a need for instrumentation, communications and procedures to use the information derived from on-line monitoring and reliability analysis, and for training the utilities' personnel, particularly those in control centres.

\section{Conclusion}

We still do not know when the next extreme geomagnetic storm will arrive, but the research so far has provided two key results to supplement the work of others:

The processes from GMD to power system failure can be formulated in a large but coherent model to analyse the complexity and manage the uncertainty; and

The improved understanding allows better responses to the threats of severe and extreme geomagnetic storms to be planned in advance and in ways that were not envisaged in March 1989 and October 2003.

Research of this nature cannot prevent severe solar storms affecting power systems, but can help in mitigating and managing the outcomes more effectively.

Acknowledgements. Many organisations have contributed essential funding for the research, including the South African national electricity utility Eskom through contracts and its Tertiary Education Support Programme, the National Research Foundation through its grant and THRIP programmes, collaboration funding from the BMBF in Germany and Royal Society in UK, and the University Research Committee of UCT. Participation in a TIEMS conference where a draft of the paper was presented was supported by NVE and Statnett. Key research collaborators include scientists, engineers and students at the South African National Space Agency (formerly known as Hermanus Magnetic Observatory), BGS Edinburgh, Eskom, and the Universities of Stuttgart, KTH (Stockholm) and Cape Town. Two colleagues, Dr. Pierre Cilliers (SANSA) and Dr. Ron Herman (UCT), deserve special recognition for being involved in our work from the start.

\section{References}

Andersson, G., P. Donalek, R. Farmer, N. Hatziargyriou, and I. Kamwa, Causes of the 2003 major grid blackouts in North America and Europe, and recommended means to improve system dynamic performance, IEEE Transactions on Power Systems, 20 (4), 1922-1928, 2005.

Bernhardi, E.H., T.A. Tjimbandi, P.J. Cilliers, and C.T. Gaunt, Improved calculation of geomagnetically induced currents in power networks in low-latitude regions, Power Systems Computation Conference, Glasgow, pp. 1391-1395, IBSN 978-1-61738857-6, 2008.

Dzobo, O, C.T. Gaunt, and R. Herman, Investigating the use of probability distribution functions in reliability-worth analysis of electric power systems, Int. J. Electr. Power Energy Syst., 37 (1), 110-116, DOI: 10.1016/j.ijepes.2011.12.013, 2012.

Edimu, M., K. Alvehag, C.T. Gaunt, and R. Herman, Analyzing the performance of a time-dependent probabilistic approach for bulk 
C.T. Gaunt: Responses for electricity utilities to severe solar storms

network reliability assessment, Electr. Power Syst. Res., 104, 156-163, DOI: 10.1016/j.epsr.2013.06.016, 2013.

Gaunt, C.T., and G. Coetzee, Transformer failures in regions incorrectly considered to have low GIC-risk. IEEE PowerTech, Lausanne, 807-812, DOI: 10.1109/PCT.2007.4538419, 2007.

Gaunt, C.T., and M. Malengret, Why we use the term non-active power, and how it can be measured under non-ideal power supply conditions, Power Engineering Society Conf. and Expo. in Africa (PowerAfrica), Johannesburg, 9-13 July 2012,

DOI: 10.1109/PowerAfrica.2012.6498641, 2012.

Kappenman, J.G., and V.D. Albertson, Bracing for the Geomagnetic Storms, IEEE Spectrum Magazine, (March), 27-33, 1990.

Liu, C.M., L.G. Liu, and R. Pirjola, Geomagnetically induced currents in the high-voltage power grid in China, IEEE Trans. Power Delivery, 24, 4, 2009.

Malengret, M., and C.T. Gaunt, General theory of average power for multi-phase systems with distortion, unbalance and direct current components, Electr. Power Syst. Res., 84, 224-230, DOI: 10.1016/j.epsr.2011.11.020, 2011.

Ministry of Power, Report of the Enquiry Committee on Grid Disturbance in Northern Region on 30th July 2012 and in Northern, Eastern \& North-Eastern Region on 31st July 2012, New Delhi, 2012.

Minnaar, U.J., C.T. Gaunt, and F. Nicolls, Characterisation of power system events on South African transmission power lines, Electr. Power Syst. Res., 88, 25-32, DOI: 10.1016/j.epsr.2012.01.015, 2012.

Molinski, T.S., Why utilities respect geomagnetically induced currents, J. Atmos. Sol. Terr. Phys., 64 (16), 1765-1778, 2002.

Moodley, N., and C.T. Gaunt, Developing a power transformer low energy degradation assessment triangle, Power Engineering
Society Conf. and Expo. in Africa (PowerAfrica), Johannesburg, 9-13 July, DOI: 10.1109/PowerAfrica.2012.6498647, 2012.

NERC, Special reliability assessment interim report: Effects of geomagnetic disturbances on the bulk power system. North American Electric Reliability Corporation, Atlanta, GA, 2012.

Oyedokun, D.T., M. Simon, and C.T. Gaunt, Introduction of a more detailed calculation of geomagnetically induced currents in transmission networks, Proc. of Southern African Universities' Power Engineering Conference, Potchefstroom, pp. 211-215, 2013.

Oyedokun, D.T., C.T. Gaunt, S. Tenbohlen, M. Heindl, M. Beltle, M. Reuter, and D. Schneider, Laboratory test for GIC effects on power transformers, XVII International Symposium on High Voltage Engineering, VDE-Verlag, Berlin, Offenbach, 2011.

Pulkkinen, A., E. Bernabeu, J. Eichner, C. Beggan, and A.W.P. Thomson, Generation of 100-year geomagnetically induced current scenarios, Space Weather, 10, S04003, DOI: 10.1029/2011SW000750, 2012

Thomson, A.W.P., E.B. Dawson, and S.J. Reay, Quantifying extreme behavior in geomagnetic activity, Space Weather, 9, S10001, DOI: 10.1029/2011SW000696, 2011.

Thomson, A.W.P., C.T. Gaunt, P. Cilliers, J.A. Wild, B. Opperman, L.-A. McKinnell, P. Kotze, C.M. Ngwira, and S.I. Lotz, Present day challenges in understanding the geomagnetic hazard to national power grids, Adv. Space Res., 45 (9), 1182-1190, 2010.

Torta, J.M., L. Serrano, J.R. Regué, A.M. Sánchez, and E. Roldán, Geomagnetically induced currents in a power grid of Northeastern Spain, Space Weather, 10, S06002,

DOI: 10.1029/2012SW000793, 2012.

Cite this article as: Gaunt C.T: Reducing uncertainty - responses for electricity utilities to severe solar storms. J. Space Weather Space Clim., 2014, 4, A01. 